\title{
Beam quality changes in Hermite-Gauss mode fields propagating through Gaussian apertures
}

\author{
J. Serna, P. M. Mejías, and R. Martínez-Herrero
}

The beam quality behavior of Hermite-Gauss laser modes propagating through Gaussian apertures is analyzed.

The characterization of the spatial behavior of laser profiles and the establishment of an adequate beam quality parameter constitutes a field of current interest that is important in the comparison of the performances of laser devices and in the design of laser optics. Recently 1,2 a family of measurable parameters was defined that describes a number of properties of beams under propagation through general first-order optical systems, and a new parameter was introduced in the literature for use as an appropriate description of the beam quality in passive and active media. $^{3-7}$ Moreover recently it has been shown ${ }^{8}$ that a certain class of soft-edge diffraction aperture, namely, the so-called Gaussian aperture, can suitably control the quality of Gaussian Schell-model beams. In the following, after introducing the pertinent definitions and the formalism to be employed, we analyze the quality changes in Hermite-Gauss laser modes when they propagate through Gaussian apertures.

Restricting ourselves for simplicity to essentially bidimensional beams, let us start from the amplitude of the $n$ th-order Hermite-Gauss mode:

$$
f_{n}(x)=H_{n}(x / w) \exp \left(-x^{2} / 2 w^{2}+i k x^{2} / 2 R\right),
$$

where $H_{n}$ denotes the $n$ th-order Hermite polynomial, $x$ is the spatial variable transversal to the propagation

Departamento de Óptica, Facultad de Ciencias Físicas, Universidad Complutense, 28040 Madrid, Spain.

Received 2 June 1992.

0003-6935/93/071084-03\$05.00/0.

(c) 1993 Optical Society of America. direction $z, k$ is the wave number, and $w$ and $R$ are the beamwidth and curvature radius at plane $z$.

We use here the Wigner distribution function formalism, ${ }^{9}$ taking into account now that, since the field is coherent, its cross-spectral density function $\Gamma$ is factorizable. It is well known that the Wigner distribution function is given in terms of $\Gamma$ as follows:

$$
\begin{aligned}
& h(x, u ; z) \\
& \quad=\int_{-\infty}^{+\infty} \Gamma(x+s / 2, x-s / 2 ; z) \exp (-i k u s) \mathrm{d} s,
\end{aligned}
$$

where $k u$ is the wave-vector component along the $x$ axis (consequently $u$ represents the angle of propagation without the evanescent waves being taken into account), and $P_{i}$ is the total irradiance. When we define the average $\langle q\rangle$ in the form

$$
\langle q\rangle=\left(1 / P_{i}\right) \int_{-\infty}^{+\infty} q h(x, u ; z) \mathrm{d} x \mathrm{~d} u
$$

and assume that $\langle x\rangle=\langle u\rangle=0$ (this is not a restriction since it is simply equivalent to a shift in the coordinate system), a beam quality parameter can be defined as

$$
Q=\left\langle x^{2}\right\rangle\left\langle u^{2}\right\rangle-\langle x u\rangle^{2},
$$

where the $\langle x u\rangle$ term vanishes at the beam waist. The $\left\langle x^{2}\right\rangle$ and $\left\langle u^{2}\right\rangle$ quantities are usually interpreted as the (squared) width and the (squared) far-field divergence of the beam. It is important to note that the $Q$ 
parameter is closely related to the well-known $M^{2}$ parameter ${ }^{5}$ by

$$
Q=\left(M^{2}\right)^{2} / 4 k^{2}
$$

The lower limit of $Q$ (the highest quality) can be shown to be $1 / 4 k^{2}$, which is reached only by pure Gaussian beams.

Let us now consider that at plane $z$ the $n$ th-order Hermite-Gauss laser beam given in Eq. (1) passes through a centered Gaussian aperture $t(x)$ defined by its transmittance function:

$$
t(x)=\exp \left(-x^{2} / 2 r^{2}\right) .
$$

If we use the notation

$$
\begin{aligned}
A(\beta, n, m) & =\int_{-\infty}^{+\infty} H_{n}(x) H_{m}(x) \exp \left(-x^{2} / 2 \beta^{2}\right) \mathrm{d} x, \\
B(\beta, n) & =\int_{-\infty}^{+\infty} x^{2} H_{n}(x) H_{m}(x) \exp \left(-x^{2} / 2 \beta^{2}\right) \mathrm{d} x,
\end{aligned}
$$

and define a $\mu$ parameter as

$$
1 / \mu^{2}=1+\left(w^{2} / r^{2}\right)
$$

with $0 \leq \mu \leq 1$ [note that $\mu=0 \Leftrightarrow r=0 \Rightarrow t(x)=0$ and $\mu=1 \Leftrightarrow r=\infty \Rightarrow t(x)=1]$, then, taking into account some integrals of the Hermite functions, ${ }^{10}$ we can show that the beam-quality parameter $Q$ is given by

$$
\begin{aligned}
Q(\mu, n)= & {\left[B(\mu, n) / 2 k^{2} A^{2}(\mu, n, n)\right] } \\
& \times\left[4 n^{2} A(\mu, n-1, n-1)\right. \\
& +\left(1 / \mu^{2}\right) A(\mu, n, n) \\
& -4 n(n-1) A(\mu, n-2, n)],
\end{aligned}
$$

where the $\mu$ and $n$ dependence of the $Q$ parameter have been written explicitly. The intensity loss $P_{o} / P_{i}$ can also be found:

$$
\left(P_{o} / P_{i}\right)(\mu, n)=\left(1 / 2^{n} n ! \sqrt{\pi}\right) A(\mu, n, n),
$$

where $P_{o}$ is the output power.

In Fig. 1 are plots of the beam-quality parameter in terms of $\mu$ for the first Hermite-Gauss modes. In Fig. 2 is the quotient $P_{o} / P_{i}$ versus $\mu$. In relation to these figures it is important to note that parameter $\mu$ depends on $z$ through the quantity $w(z)$. Consequently Figs. 1 and 2 also represent the behavior of $Q$ and the intensity loss as functions of the relative position $z$ of the Gaussian aperture with respect to the beam. It is then clear that the quality will be improved when the transmittance is placed far from the waist plane of our Hermite-Gauss laser mode.

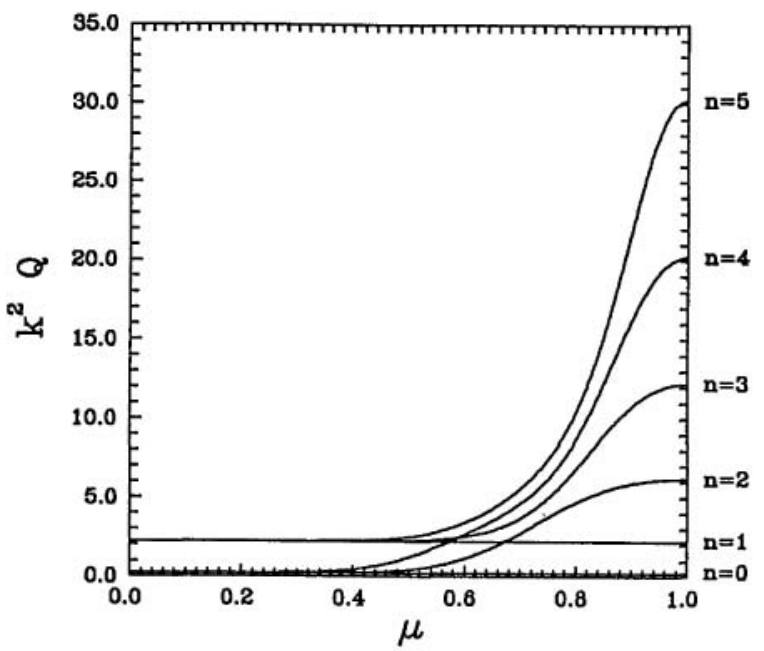

(a)

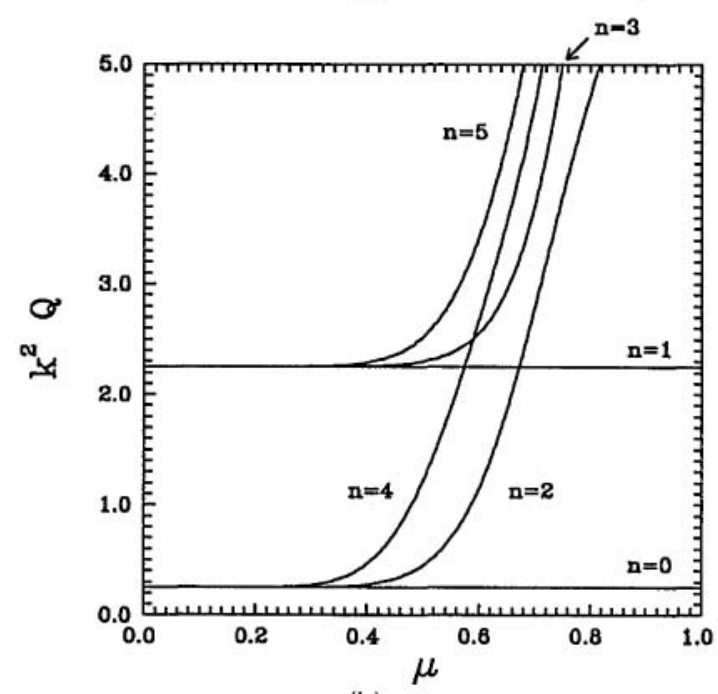

(b)

Fig. 1. (a) Beam quality parameter $Q$ versus $\mu$ for several Hermite-Gauss fields. On the ordinates we have the dimensionless parameter $k^{2} Q$. (b) Detail of (a) showing more clearly the quality behavior of small $\mu$.

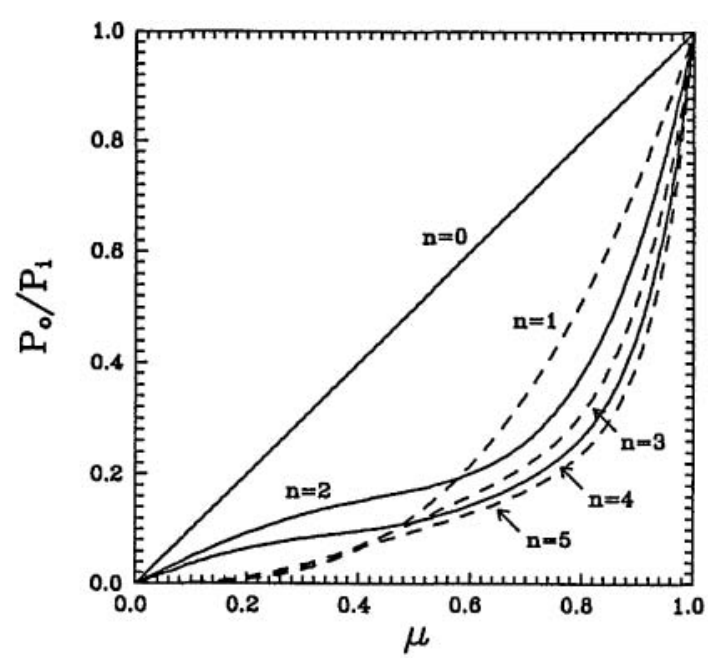

Fig. 2. Intensity loss (quotient $P_{o} / P_{i}$ ) versus $\mu$. 
From the figures discussed above three main conclusions can be drawn:

(1) The quality of both the fundamental Gaussian mode and the first-order Hermite-Gauss mode cannot be modified by means of Gaussian apertures.

(2) When $\mu \rightarrow 0$ the quality behavior of the even modes is similar to that of the fundamental mode, but it clearly differs from the behavior of the odd modes, which for small $\mu$ reaches the quality of the firstorder mode.

(3) As a rule of thumb when $\mu \rightarrow 0$ losses are smaller for even modes.

We are grateful to A. E. Siegman for proposing the problem treated in this paper. We also thank the Comisión Interministerial de Ciencia y Tecnología of Spain, under project ROB90-539, for financial support.

\section{References}

1. J. Serna, R. Martínez-Herrero, and P. M. Mejías, "Parametric characterization of general partially coherent beams propagating through ABCD optical systems," J. Opt. Soc. Am. A 8, 1096-1100 (1991).

2. J. Serna, P. M. Mejías, and R. Martínez-Herrero, "Beam quality dependence on the coherence length of Gaussian Schell-model fields propagating through $A B C D$ optical systems," J. Mod. Opt. 39, 625-635 (1992).

3. S. Lavi, R. Prochaska, and E. Keren, "Generalized beam parameters and transformation laws for partially coherent light," Appl. Opt. 27, 3696-3703 (1988).

4. R. Simon, N. Mukunda, and E. C. G. Sudarshan, "Partially coherent beams and a generalized $A B C D$ law," Opt. Commun. 65, 322-328 (1988).

5. A. E. Siegman, "New developments in laser resonators," in Optical Resonators, D. A. Holmes, ed., Proc. Soc. Photo-Opt. Instrum. Eng. 1224, 2-20 (1990).

6. R. Martínez-Herrero and P. M. Mejías, "Beam characterization through active media," Opt. Commun. 85, 162-166 (1991).

7. J. Serna, P. M. Mejías, and R. Martínez-Herrero, "Beam quality in monomode diode lasers," Opt. Quantum Electron. 24, 5881-5887 (1992).

8. J. Serna, P. M. Mejías, and R. Martínez-Herrero, "Beam quality changes of Gaussian Schell-model fields propagating through Gaussian apertures," Appl. Opt. 31, 4330-4332 (1992).

9. M. J. Bastiaans, "Application of the Wigner distribution function to partially coherent light," J. Opt. Soc. Am. A 3, 1227-1246 (1986).

10. I. S. Gradshteyn and I. M. Ryzhik, Table of Integrals, Series and Products (Academic, New York, 1965). 